\title{
METHOD OF COMPREHENSIVE ASSESSMENT OF EFFICIENCY OF DECREASE OF FIRE AND EXPLOSION RISK OF COAL SUSPENDED PARTICLES
}

\author{
Vladimir Arkhipov ${ }^{1, *}$, Yuriy Patrakov ${ }^{2}$, Aleksandr $\operatorname{Sechin}^{3}$, Andrey Sechin $^{3}$, and \\ Anna Usanina ${ }^{2}$ \\ ${ }^{1}$ National Research Tomsk State University, 634050 Tomsk, Russia \\ ${ }^{2}$ Federal Research Center for Coal and Coal Chemistry of Siberian Branch of the Russian Academy of \\ Sciences, 650610 Kemerovo, Russia \\ ${ }^{3}$ National Research Tomsk Polytechnic University, 634050 Tomsk, Russia
}

\begin{abstract}
Methods and results of experimental study of explosive and wetting characteristics of coal dust are presented. Modified evaluation method of explosive characteristics of coal dust allows estimating critical concentrations of suspended dust at variation of temperature of ionizing ignition source corresponding to beginning of flame propagation by means of approximation method. Method of measuring the contact angle of particles of coal powder is based on visualization of the drop placed on the pressed coal.
\end{abstract}

\section{Introduction}

Coal dust is an inevitable element of practically all technological stages of production, transportation, preparation, and processing of coal. This determines the increased fire and explosion risk of enterprises of coal industry and energetics [1,2]. Inflammation of the dust-air mixture is a multivariable process so accepted classifications and characteristics of coal dust are quite relative and does not take into account majority of factors that can ensure trouble-free operation of such enterprises.

One of the most effective ways to reduce the explosion of suspended coal particles is irrigation of mine face using drop-liquid aerosol. This reduces the risk of self-ignition of coal dust. It is necessary to conduct the complex experiments on concentration limits of flame propagation for dry and wetted coals from different deposits to evaluate the effect of the wettability of particular coal ranks on their explosiveness. In this regard, the development of new devices and methods for studying the wetting properties of the coal surface and critical conditions of the flame propagation in aerosuspension are still relevant since only a deep understanding of the combustion and coal particles wetting processes can give the effective technological decisions that reduces the likelihood of coal dust explosions.

In this work the methods of experimental study of fire and explosion dangerous and wetting characteristics of coal particles are presented.

\footnotetext{
*Corresponding author: leva@niipmm.tsu.ru
} 


\section{Experimental study of fire and explosion dangerous characteristics of coal suspended particles}

Modified method of successive iteration is proposed to study the fire and explosion dangerous characteristics of coal dust. This method allows estimating the critical concentrations of suspended dust particles corresponding to the beginning of flame propagation at variation of temperature of ionizing ignition source.

Scheme of experimental setup for studying the fire and explosion dangerous characteristics of particles suspended in air is shown in fig. 1. The setup consists of test chamber 1 , round spray in coincidence with nozzle 2 , electromagnetic valve 3 , receiver 4 , manometer 5, strain-gage pressure sensor 6 , valves $7,8,15,16$, thermocouple sensors 9,10 , strain gauge scanner recorder with oscilloscope 11 , multichannel recorder 12, ignition source 13, compressor 14, forming unit of gas medium 17.

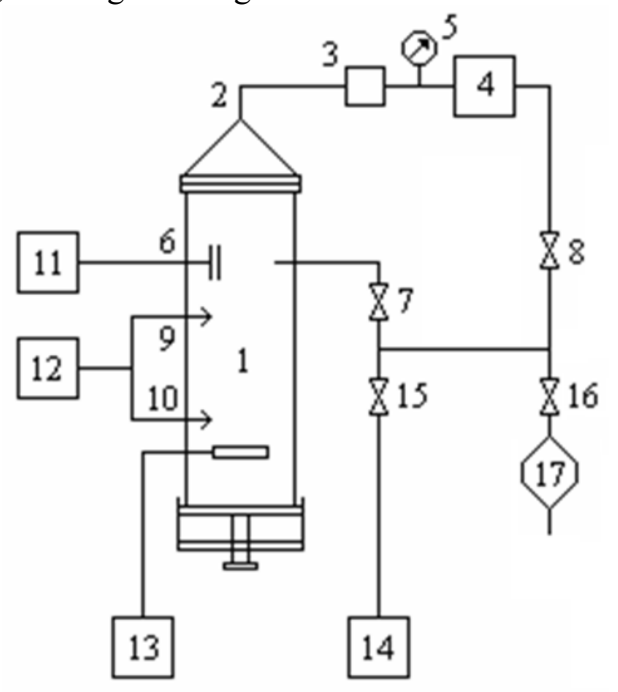

Fig. 1. Scheme of experimental setup for studying the critical conditions of flame propagation in cloud of suspended particles.

In previously sealed reactor chamber 1 with initial manometric pressure $p=50.7 \mathrm{kPa}$ in the receiver 4 and at driven recording units and initiating ignition source 13 the control experiment is conducted. The temperature of initiating ignition source 13 is $1050{ }^{\circ} \mathrm{C}$. In future, the data obtained is used for comparison with results of work experiments.

Then, the substance studied weighing 0.05 gram is placed in the nozzle 2 , the air pressure $p=50.7 \mathrm{kPa}$ in the receiver is adjusted, the registration is beginning, and the setup is actuated.

If the flame spreads up to the top of the thermocouple and higher and there was the pressure increase that is different from blank experiment, the experiment is considered to be a success. Otherwise or in the case of absence of one of the indicators (flame spreading to the upper flange of the chamber 1 or the absence of pressure rise) the experiment is considered to be a failure.

Exposing the nozzle, it is checked whether all the substance take part in the spraying. If the nozzle remains non-atomized substance the experiment is repeated. At that the atomizing impulse value is increased by $10.1 \mathrm{kPa}$ until the nozzle will not remain empty. If the nozzle is empty the experiment is repeated with the substance of previous mass, but atomizing impulse value is decreased on $10.1 \mathrm{kPa}$. This is the way to determine the optimal atomizing impulse for concrete substance of specified mass. 
If the ignition and flame spread is not detected, the mass of substance is increased by $0.05 \mathrm{~g}$. After positive result at given mass of the substance, the limiting conditions of flame spread increasing and decreasing the mass of the substance by $0.01 \mathrm{~g}$ is searched. This procedure remains until two dust concentrations that are different no more than on $0.01 \mathrm{~g}$ will be found. The first concentration corresponds to ignition occurring, the second one corresponds to ignition rejection. The presence or absence of ignition at these concentrations is examined by means of more than ten control experiments with the mass of the substance that does not give the ignition. In the case of ignition during the control experiments the mass of the substance is decreased by $0.01 \mathrm{~g}$ until the entire series of noncombustible suspended particles will be obtained.

After each experiment the bottom flange is removed by means of cutoff of the ignition source 13 , the reaction chamber 1 is cleaned of the settled dust and combustion products and purged with compressed air.

Defining the burning area of suspended dust particles at the change of temperature of initiating ignition source, the experiment is carried out for the above-mentioned technique. The ignition source temperature is reduced by $50{ }^{\circ} \mathrm{C}$ until the suspended dust particles ignite and burning spreads throughout the volume of the reaction chamber.

Testing of the method and experimental setup for studying the critical conditions of flame propagation in air-dust mixture is conducted using the benzoic acid. For particles with an equivalent diameter of $d_{p}=(160 \div 200) \mu \mathrm{m}$, lower concentration limit of flame spread is equal to $C=36.7 \mathrm{~g} / \mathrm{m}^{3}$ at the initiating source temperature of $1100{ }^{\circ} \mathrm{C}$. This is in good agreement with literature data [3]. Thus, the proposed experimental method is not only consistent with the standard one [4], but also applicable for expanding the fields of its application.

Effect of the initiating ignition source temperature was studied on the sample of Kuznetsk coal rank KO $(0 \div 50 \mathrm{~mm})$. From the results presented (fig. 2) it is seen that airdust particles are more sensitive to initiation by the source with a high ignition temperature.

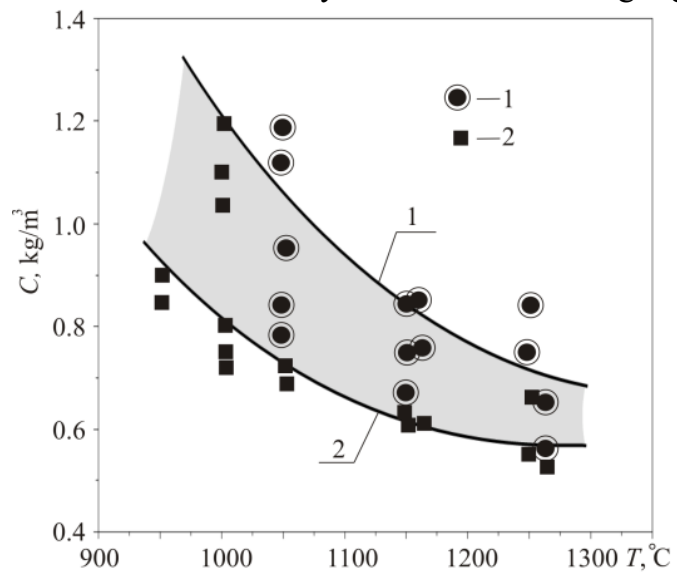

Fig. 2. Concentration dependence of coal dust burning field on ignition source temperature: 1 burning with marked increase in pressure; 2 - burning without marked increase in pressure in testing chamber.

Analysis of the flame-front spread has been shown that the burning of coal dust up to some concentrations is characterized by weak growth of manometric pressure in the reaction chamber. In a similar way to burning of hydrocarbon blend with air [5-7] it is possible to suggest that for coal dust the regime of "coal" flame exists. The transition of the burning front to "hot" flames area (i.e. with a marked increase in pressure) occurs for 
the coal sample at increasing the concentration of air-dust mixture in $(100 \div 150) \mathrm{g} / \mathrm{m}^{3}$ and more, depending on the source ignition temperature. For example, the concentration of coal dust that characterizes the burning process of "cold" flame is approximately $750 \pm 50 \mathrm{~g} / \mathrm{m}^{3}$ at the temperature of initiating ignition source $1150{ }^{\circ} \mathrm{C}$ (the lower curve in fig. 2). But concentration characterizing the burning process in the "hot" flame regime is equal to $850 \pm 50 \mathrm{~g} / \mathrm{m}^{3}$ at the same temperature (upper curve, fig. 2).

In this case the mechanism of flame distribution on air-dust mixture is significantly different from the gas phase of "cold" flame of "ideal" systems and it can be simply interpreted as a "relay" transfer of the thermal pulse from one coal particle to another. And so it will be determined by larger number of parameters (dispersion of dust particles, oxygen concentration and thermal conductivity of gas phase, mechanism and rate of burning of the coal particle and others).

Thus, conducting studies on the standard method [4] it is impossible to change the temperature of the ignition source and this limits the understanding about the ignition process.

\section{Experimental study of wetting characteristics of coal suspended particles}

The study of the wettability of coal powder particles was conducted on the setup consisting of the matrix for pressing 1 , the sample pressed 2, a device for droplets forming 3 , highspeed camera 4, and drop 5 located on the coal powder sample pressed (fig. 3). The essence of the method for determining the coal powder wettability is to directly measure the contact angle of the drop located on the pressed coal particles briquette of cylindrical shape [8]. The problem of liquid penetration through the sample pressed is solved by means of preparing the briquette of specified highness and diameter, pressing at specified pressure in matrix of special form, and previous preparation of powder sample with specified particle size.

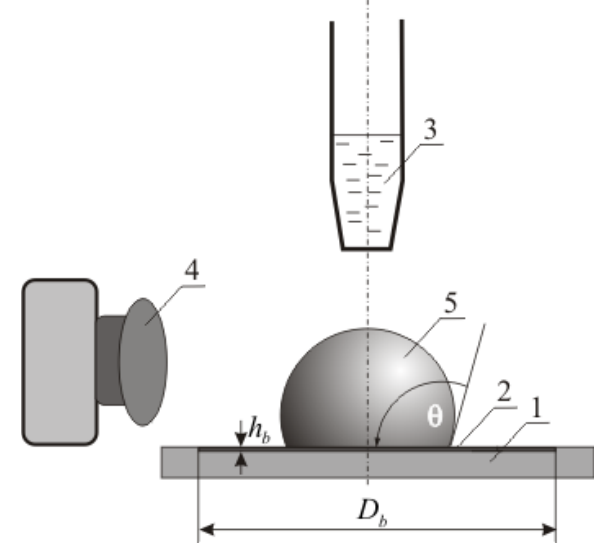

Fig. 3. Scheme of experimental setup for determining the wettability of coal powder.

It is necessary to provide the corresponding briquette diameter $D_{b}$ since it is not known how liquid drop behaves on the surface of pressed briquette (it spreads in a thin liquid layer or forms the drop on it). The experiments for wetting case closed to full wetting performed for drop of silicon oil (PDMS-100) spreading on acrylic glass has shown that the maximum value of drop contact radius is equal to $r_{k} \cong 4 D$. The briquette diameter is chosen from 
the condition $D_{b} \leq 2 D$ that provides the location of the drop on the briquette surface and possibility of measuring the contact angle.

Evaluation experiments in choice of optimal height of the briquette $h_{b}$ have shown that at value $h_{b}=0.1 D$ the uniformly height-distributed briquette can be obtained. For smaller values of the height $h_{b}$ it is not enough the required weight of powder for the formation of tablets.

Experiments for coal samples have shown that more effective value of compacting pressure is equal to $p>400 \mathrm{MPa}$. Choice of dispersivity of initial powder with maximum particle size no more than $100 \mu \mathrm{m}$ and compacting pressure no more than $400 \mathrm{MPa}$ allows decreasing the porosity of the sample significantly, obtaining the plane working surface and the stable drop on a plane surface.

Since the height of the briquette is low there is the possibility of the briquette destruction during their ejecting from the press mold. It is suggested to carry out the pressing of powder particles in the matrix to avoid the briquette destruction. The size of matrix corresponds to the required values of briquette diameter and height (Fig. 4). The contact angle measurement is performed on the briquette that is placed in the matrix for pressing.

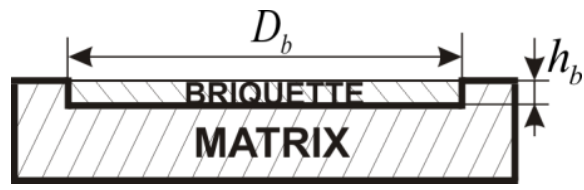

Fig. 4. Matrix for pressing of powder particles.

For low-viscosity liquids after their impact with the solid surface the decaying oscillations of contact angle and the drop height are observed. The less the impact velocity is, the lower the oscillation frequency is and the faster equilibrium drop shape is formed. Since it is difficult to provide the zero impact velocity at placing the liquid drop on a pressed sample it should be ensured the lowest impact velocity.

The experiments on liquid drop spreading on solid surface showed that average value of minimal impact velocity that can be provided is equal to $1 \mathrm{sm} / \mathrm{s}$. It is shown that time of forming the equilibrium contact angle of the distilled water drop at $u=1 \mathrm{sm} / \mathrm{s}$ is equal to $t=200 \mathrm{~ms}$. This value is lower limit of the time that corresponds to beginning of measurement of equilibrium contact angle. The choice of distilled water as an etalon liquid is described the following arguments. Firstly, it is more widely used liquid for the determining the powder wettability, and, secondly, for viscous liquid the value $t$ will be more.

Necessity of decreasing the time of forming the equilibrium drop shape exists due to the fact that it is observed the evaporation process of the drop along with formation of equilibrium contact angle of the drop. The process of evaporation results in the change of gas phase (air is changed on saturated steam) and the contact angle value is changed.

In the work, the analysis of evaporation degree of the distilled water drop during their spreading on acrylic surface at room temperature was performed. The choice of this liquid is described the fact that it is one of volatile liquids. Calculation of the drop volume by means of processing the video for different time interval $t_{i}$ was conducted to evaluate the evaporation degree:

$$
V_{k}\left(t_{i}\right)=2 \pi \int_{0}^{r_{k}\left(t_{i}\right)} r h(r) d r,
$$


where $V_{k}\left(t_{i}\right)$ is the drop volume in specific time $t_{i} ; h(r)$ is the dependence of the drop height on radial coordinate for time moment $t=t_{i} ; r_{k}\left(t_{i}\right)$ is the contact radius in specific time. Processing of the video sequence showed that marked evaporation of the drop (deviation of $V\left(t_{i}\right)$ from initial drop volume $V=\pi D^{3} / 6$ on $0.5 \%$ ) begins at $t_{i} \sim 6 \mathrm{~s}$. This time $t$ can be considered as lower limit corresponding to the beginning of effect of physical and chemical processes, namely evaporation, on the drop. So the measurement of the contact angle must be performed in time interval $t=(0.2 \div 6.0) \mathrm{s}$ after placing the drop on the pressed sample.

Approbation of the proposed method for determining the wettability of coal powders was conducted for coal of different deposits. The results of determining the contact angle for different coal sample are presented in Table 1.

Table 1. Contact angle values $\theta$ for coal surface.

\begin{tabular}{|c|c|c|}
\hline Sample & Contact angle, degree & $\delta, \%$ \\
\hline A1 & $90.1 \pm 0.3$ & 0.3 \\
\hline A2 & $53 \pm 10$ & 18.9 \\
\hline A3 & $61 \pm 7$ & 11.4 \\
\hline A4 & $67 \pm 3$ & 4.5 \\
\hline A5 & $60 \pm 3$ & 5.0 \\
\hline A6 & $87 \pm 2$ & 2.3 \\
\hline A7 & $91 \pm 7$ & 7.7 \\
\hline A8 & $91 \pm 7$ & 7.7 \\
\hline A9 & $80 \pm 2$ & 2.5 \\
\hline
\end{tabular}

The analysis of effect of chemical composition of coals on contact angle value showed that while the mass fraction of oxygen, nitrogen and sulfur $\beta=\mathrm{O}+\mathrm{N}+\mathrm{S}$ rises, the contact angle value is decreased (fig. 5).

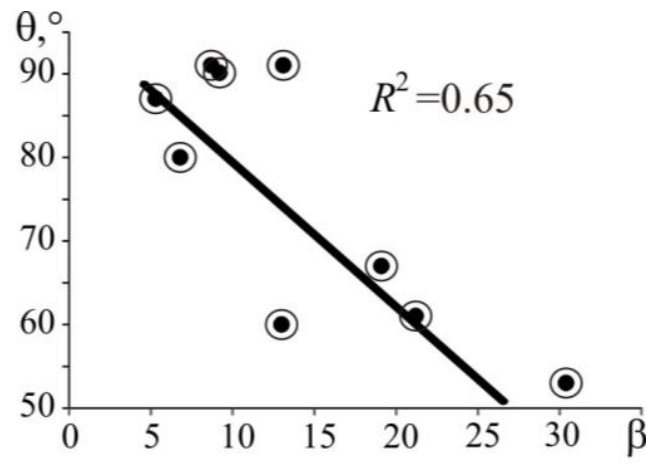

Fig. 5. Dependence of contact angle wettability of coal powders on parameter $\beta$.

The approximation curve is shown in fig. 5 . The curve with coefficient of determination $\mathrm{R}^{2}=0.65$ is obtained by means of least-squares method.

\section{Conclusion}

The modified method for studying the fire and explosion dangerous characteristics of coal mixtures and method for determining the contact angle of coal powders are presented. 
- Experimental method for studying the fire and explosion dangerous characteristics provides the determination of critical conditions of flame spread in the conditions of uniform distribution dust cloud at varying the temperature of initiating ignition source. This allows conducting more accurate substance rating and so development of measures on fire and explosion risk.

- The possibility of occurrence of "cold" flame at ignition of air coal dust mixture and its transition in "hot" flame at increasing the dust concentration has been shown. This is important for theory of fire safety engineering.

- The method for studying the wetting characteristics of coal powders has some advantages. Direct measurement of contact angle excludes the error accumulation due to indirect determination. Plane surface of the sample excludes the error of determination of contact angle due to their inequality and roughness. Reducing the liquid penetration provides the stable drop on the sample surface that allows measuring its contact angle. The method excludes the contact angle determination error occurring due to the change of gas phase composition at the drop evaporation.

- The considered methods for studying the characteristics of coal can be used for development of new devices and technological decisions in mining works.

\section{References}

1. S.B. Romanchenko, Yu.F. Rudenko, V.N. Kosterenko, Dust dynamics in coal mines (Gornoe delo, Moscow, 2011)

2. V.V. Pomerancev, S.L. Shagalova, V.A. Reznik et al., Spontaneous combustion and explosions of natural fuels dust (Energiay, Leningrad, 1978)

3. A.N. Baratov, A.Ya. Korolchenko, N.G. Kravchuk et al., Fire and explosion unsafety of substances and materials and their extinguishing agent (Khimiya, Moscow, 1990)

4. GOST 12.1.044-89. OSSS, Fire and explosion unsafety of substances and materials. Nomenclature of indicates and methods of their determination (Izdatelstvo standartov, 1965).

5. V.Ya. Bonner, C.F. Tipper, Int. 10th Symp. Combust. 145 (1965)

6. J.A. Heand Schneider, C. Volanski, Revue Roumaine de Chimie 18, 195 (1973)

7. J. A. Barnard, B. A. Harwood, Combust. Flame 21, 354 (1973)

8. V.A. Arkhipov, D.Yu. Paleev, V.F. Trofimov, A.S. Usanina, Patent RF no. 2457464, IPC G01N 13/00. Method for determining the wettability of powder materials (Publ. 27.07.2012, Bull. no. 20) 